\title{
Nonlinearity Tolerant LUT-based Probabilistic Shaping for Extended-Reach Single-Span Links
}

\author{
Pavel Skvortcov, Student Member, IEEE, Ian Phillips, Member, IEEE, Wladek Forysiak, Member, IEEE, \\ Toshiaki Koike-Akino, Senior Member, IEEE, Keisuke Kojima, Senior Member, IEEE, \\ Kieran Parsons, Senior Member, IEEE, and David S. Millar, Member, IEEE
}

\begin{abstract}
We propose Huffman-coded sphere shaping (HCSS) as a method for probabilistic constellation shaping which provides improved tolerance to fiber nonlinearities in single-span links. An implementation of this algorithm based on look-up-tables (LUTs) allows for low-complexity, multiplier-free shaping. The advantage of short-length shaping for mitigating fiber nonlinear impairments is experimentally demonstrated for a system employing dual-polarization 64-ary quadrature amplitude modulation (DP64QAM) at $56 \mathrm{GBd}$ and operating over $210 \mathrm{~km}$ of standard single-mode fiber (SSMF). A gain in achievable information rate (AIR) of 0.4 bits/4D-symbol compared with uniform signaling is measured, corresponding to a $100 \%$ improvement in shaping gain compared with ideal Maxwell-Boltzmann (MB) shaping. The combinatorial mapping and demapping algorithms can be implemented with integer addition and comparison operations only, utilizing an LUT with 100 kbit size.
\end{abstract}

Index Terms-Probabilistic shaping, sphere shaping, nonlinear fiber channel, multiplier-free LUT, experimental validation.

\section{INTRODUCTION}

$\mathbf{I}$ $\mathrm{N}$ recent years, market demand has shifted the nexus of coherent optical research toward short distance metro applications, primarily due to cloud and inter-data-center traffic. Single-span links over $10-120 \mathrm{~km}$ at high line rates have become a significant driver of bandwidth demand. Following the successful introduction of the 400G ZR standard [1], there is a great deal of interest in increasing reach and bit rate in systems that maintain low power, low latency and compatibility with pluggable optics. Concurrently, probabilistic shaping has evolved from a topic of academic interest [2] to realizable technology [3], [4]. The drivers of this adoption have been partly increased noise tolerance and partly the increased rate flexibility that can be provided by tuning the rate of the shaping mapper, while maintaining constant symbol and code rates [5]. More recently, short block-length shaping [6], [7], low-complexity shaping [8], [9] and nonlinearity mitigating shaping architectures [10] have become active research topics.

The effect of shaping length on effective signal-to-noise ratio (SNR) and achievable information rate (AIR) has been intensively studied in the literature. Infinite-length probabilistic shaping based on the Maxwell-Boltzmann (MB) distribution which can be capacity achieving in the additive white Gaussian noise (AWGN) channel - can adversely enhance the fiber

P. Skvortcov, I. Phillips, W. Forysiak are with Aston University, Birmingham, B4 7ET, UK. E-mails: \{skvortcp, i.phillips, w.forysiak\}@aston.ac.uk.

D. S. Millar, T. Koike-Akino, K. Kojima and K. Parsons are with Mitsubish Electric Research Laboratories (MERL), Cambridge, MA 02139, USA. Emails: \{millar, koike, kojima, parsons\}@merl.com. Copyright (c) 2020 IEEE nonlinearities in long-haul transmission [11] and unrepeated transmission [12]. The advantage of short-length probabilistic shaping for the nonlinear channel was thus investigated for multi-span long-haul links using constant composition distribution matching (CCDM) [13], [14] and enumerative sphere shaping (ESS) [15], [16]. Furthermore, significant shaping gain exceeding the theoretical gain for the linear channel was demonstrated for single-span links by optimally combining linear and nonlinear shaping gain contributions using shell mapping with very short shaping length [17].

Huffman-coded sphere shaping (HCSS) was recently proposed as an algorithm for a finite-length probabilistic shaping architecture [18]. The HCSS architecture approximates the optimal sphere bound [19] and provides good power efficiency with low rate loss for a given shaping sequence length. This architecture is amenable to implementation with reducedcomplexity CCDM mapping algorithms such as subset ranking (SR) and multiset ranking (MR) [20].

In this work, we experimentally compare three shaping schemes employing dual-polarization 64-ary quadrature amplitude modulation (DP-64QAM) in an extended-reach singlespan link: uniform signaling, shaping with an ideal MB distribution and HCSS. We used short shaping sequence lengths for HCSS such that the decrease in power efficiency compared with infinite-length MB shaping is more than offset by the increase in signal-to-noise ratio (SNR) in the nonlinear transmission regimes [17]. This allows us to achieve performance better than that of MB shaping, at a sequence length suitable for implementation with look-up-tables (LUTs) for storage of pre-computed multinomial coefficients.

\section{HuffMAn-Coded Sphere ShaPIng (HCSS)}

The sphere bound utilizes all constellation points for signaling on a specified multi-dimensional lattice satisfying a maximum energy constraint. By definition, this scheme achieves the best possible energy efficiency for a given rate (i.e. number of constellation points), lattice (e.g. the square lattice modulation of uniform QAM), and dimension (i.e. sequence length) [19]. Huffman-coded sphere shaping restricts the number of constellation points utilized for each unique composition to be a power of two, and then introduces a minimal number of additional compositions with higher power to ensure a dyadic distribution of compositions [18]. This enables the use of a variable length binary prefix to uniquely address compositions in the shaping architecture. The 


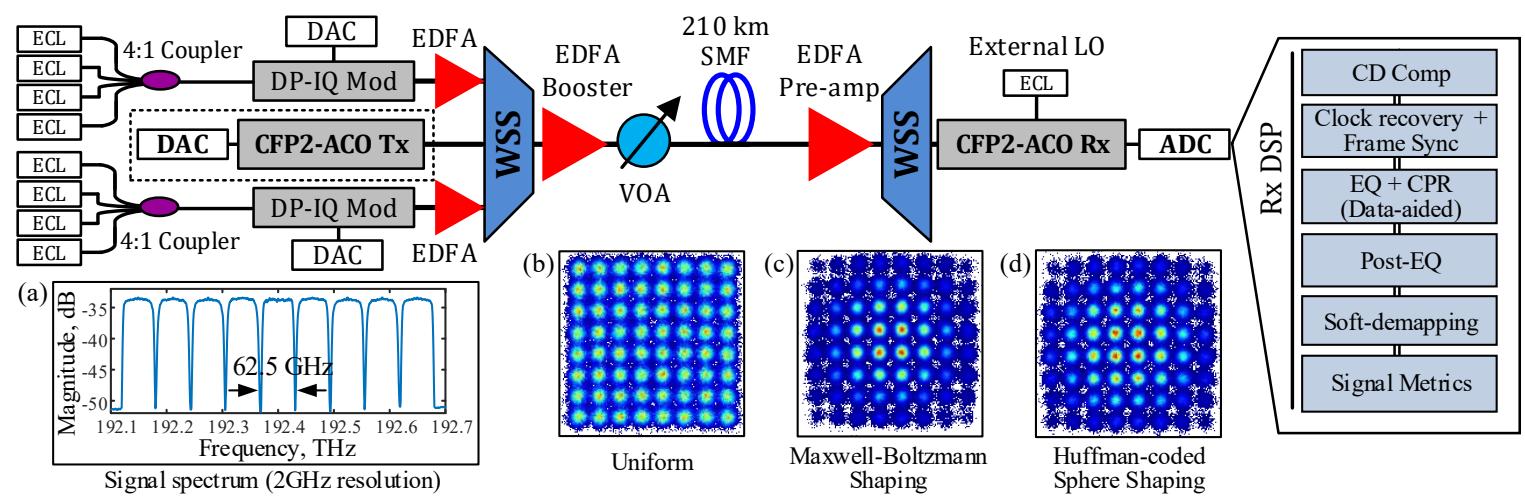

Fig. 1. Transmission experimental setup. Insets: (a) Signal spectrum (2 GHz resolution), (b) Uniform constellation, (c) Maxwell-Boltzmann shaped constellation, (d) Huffman-coded sphere shaped constellation at $L=32$.

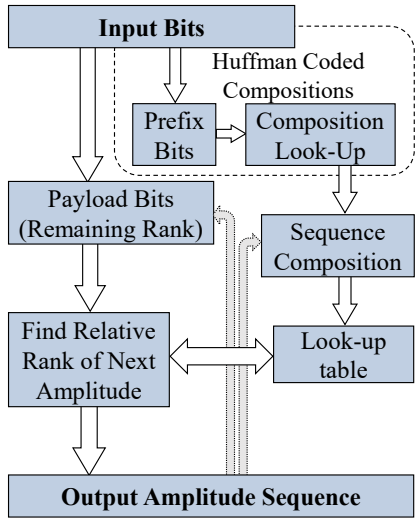

(a) HCSS Mapper

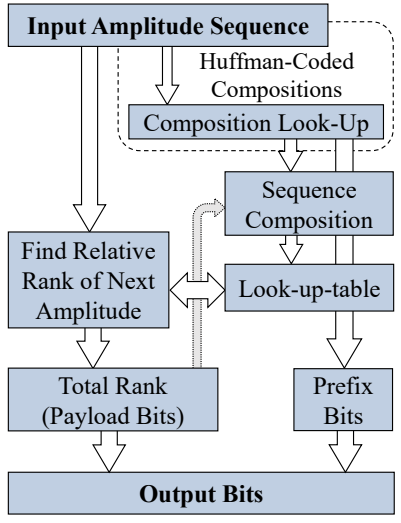

(b) HCSS Demapper
Fig. 2. Block diagram of HCSS algorithm using multiset ranking and LUT.

remaining payload bits in the binary input word are then used to address a unique permutation of the specified composition. If the MR algorithm is used as described in [18], [20], the lexicographical rank of the selected sequence corresponds to the payload bits. Additionally, we note that sequence ranks may be computed without multiplication operations by precomputing multinomial coefficients and storing them in an LUT. Therefore, both mapping in Fig. 2(a) and demapping in Fig. 2(b) are performed iteratively on a per symbol basis, using LUTs, and integer comparison and addition operations.

To investigate system performance employing short-length HCSS in comparison with uniform signaling and infinitelength MB shaping, three HCSS shapers were considered - each with shaping sequence lengths of $L=16,32,48$ amplitudes at a shaping rate of $R_{\mathrm{S}}=1.75$ bits/amplitude (with the corresponding number of compositions used: 275, 1293, 3548 ). The LUT size required for $L=16,32,48$ is approximately $30 \mathrm{kbit}, 100 \mathrm{kbit}$ and $1 \mathrm{Mbit}$, respectively [20]. We note that a granularity in shaping rates of $1 / L$ bits/amplitude can be achieved, and LUT size does not change with shaping rate, but only with shaping sequence length.

In all cases, each 4 consecutive amplitudes from a single shaped sequence were mapped onto the 4 simultaneous quadratures of the modulated 4D-symbols of the DP64QAM format. This approach results in a 4D amplitude distribution. Hence, rate loss is calculated in $\mathrm{b} / 4 \mathrm{D}$ as $R_{\text {loss }}=$
$\mathrm{H}(X)-D \cdot\left(R_{\mathrm{S}}+1\right)$, where $\mathrm{H}(X)$ is the entropy of $4 \mathrm{D}$ symbols $X, D$ accounts for mapping dimensionality $(D=4)$ and " 1 " accounts for the sign bit per dimension. This 4D mapping effectively shortens the length of the shaped 4Dsymbol sequence in the time-domain, compared with a 1D or 2D mapping [14], without increase in the rate loss.

For the MB shaping case, signals were drawn from an MB distribution with the entropy of 1.75 bits/amplitude [11]. We note that symbols in the transmitted signal were drawn independently and identically on the underlying probability mass function (PMF). This method may be considered to give a finite-length sample of an infinite-length shaped sequence, which incurs no rate loss.

\section{TRANSMISSION EXPERIMENT}

\section{A. Experimental setup}

The experimental setup is shown in Fig. 1. Transmission of 9 channels modulated by DP-64QAM (with root-raised cosine pulse shaping with $10 \%$ roll-off factor) and operating at $56 \mathrm{GBd}$ on a $62.5 \mathrm{GHz}$ grid was carried out over a $210 \mathrm{~km}$ single-span link of SSMF (SMF-28 with approximate parameters at $1550 \mathrm{~nm}$ : dispersion, $17 \mathrm{ps} / \mathrm{nm} / \mathrm{km}$; attenuation, $0.19 \mathrm{~dB} / \mathrm{km}$; nonlinear coefficient, $1.3 / \mathrm{W} / \mathrm{km}$ ). All channels used the same shaping scheme under investigation. The signal spectrum is shown in inset (a) of Fig. 1.

The central channel under test (CUT) was generated using a pluggable CFP2 analog coherent optics (ACO) transceiver (integrated laser with $100 \mathrm{kHz}$ linewidth) and a $92 \mathrm{GSa} / \mathrm{s}$ digital-to-analog converter (DAC). Eight interfering channels were divided into two groups of 4 channels and generated by two pairs of DP Mach-Zehnder in-phase/quadrature (IQ) modulators and DACs, where 4 external cavity lasers with 100 $\mathrm{kHz}$ linewidth combined with a polarization maintaining coupler were used for each modulator/DAC pair. The two groups of interfering channels were then pre-amplified by erbiumdoped fiber amplifiers (EDFAs), and spectrally interleaved and combined with the CUT via a programmable wavelength selective switch (WSS).

At the input of the link a booster EDFA and variable optical attenuator (VOA) were used to control the total launched power. After transmission, the signal was pre-amplified by an EDFA with a noise figure of $5.5 \mathrm{~dB}$, then the CUT was 
filtered by a WSS and received by a CFP2-ACO transceiver (an external laser with $100 \mathrm{kHz}$ linewidth was used as a local oscillator) followed by a $92 \mathrm{GSa} / \mathrm{s}$ analog-to-digital converter (ADC). For back-to-back (BtB) test, the output of transmitterside WSS was directly connected to the receiver-side WSS.

Digital signal processing (DSP) was performed offline according to the generic flow in Fig. 1 as follows: chromatic dispersion was compensated, clock recovery was performed by a frequency-domain Gardner algorithm, conventional complexvalued decision-directed least-mean squares (DD-LMS) $2 \times 2$ multiple-input multiple-output (MIMO) equalization was performed in conjunction with carrier phase recovery (CPR) in fully data-aided mode, and post-equalization was done using real-valued DD-LMS $2 \times 2$ MIMO equalizers to enable compensation of residual transmitter IQ impairments [21]. Softdemapping assumed a circularly symmetric Gaussian channel [11], and transmission performance metrics were averaged over approximately $5 \times 10^{6}$ symbols.

Constellation diagrams for uniform signaling, MB shaping and HCSS (at $L=32$ ) in BtB are shown in insets (b)-(d) of Fig. 1. We note that no significant visual difference can be observed between HCSS and MB shaped constellations.

\section{B. Performance metrics}

AIR for bit-metric decoding (BMD) impacted by the rate loss associated with finite-length shaping scheme [6] is calculated in $\mathrm{b} / 4 \mathrm{D}$ as

$$
\mathrm{AIR}=\left[\mathrm{H}(X)-\sum_{i=1}^{m} \mathrm{H}\left(B_{i} \mid Y\right)\right]-R_{\mathrm{loss}},
$$

where $X$ are the transmitted symbols, $Y$ are the received symbols after signal recovery algorithms, $B_{i}(i=1, \ldots, m)$ are the binary labels. For uniform signaling and infinite-length MB shaping, we have $R_{\text {loss }}=0$ and, hence, the AIR is given by the generalized mutual information [22].

Effective SNR [11] is estimated as

$$
\mathrm{SNR}_{\mathrm{eff}}=\frac{\operatorname{Var}\left[X^{\prime}\right]}{\operatorname{Var}\left[Y-X^{\prime}\right]},
$$

where $\operatorname{Var}[\cdot]$ denotes the variance, and $X^{\prime}$ are the adjusted transmitted symbols, such that constellation points $x_{i}^{\prime}(i=$ $1, \ldots, M)$ represent centroids of corresponding received symbols. We note that effective SNR accounts for both linear and nonlinear noise contributions, including transceiver noise floor.

\section{RESUlts}

\section{A. Back-to-back}

Fig. 3 shows a BtB characterization of the system under consideration. Fig. 3(a) shows AIR as a function of optical SNR (OSNR). Infinite-length MB shaping achieves superior performance with a $0.48 \mathrm{~b} / 4 \mathrm{D}$ AIR gain compared with uniform DP-64QAM over an operating SNR range of 11$13 \mathrm{~dB}$. HCSS shaping gains are $0.32,0.22,0.08 \mathrm{~b} / 4 \mathrm{D}$ for $L=48,32,16$, respectively. From Fig. 3(b) we conclude that the difference in the effective SNR is negligible between uniform and shaped signals, indicating that the shaping algorithms do not introduce a significant implementation penalty in the operating SNR region of interest and with chosen shaping rate.

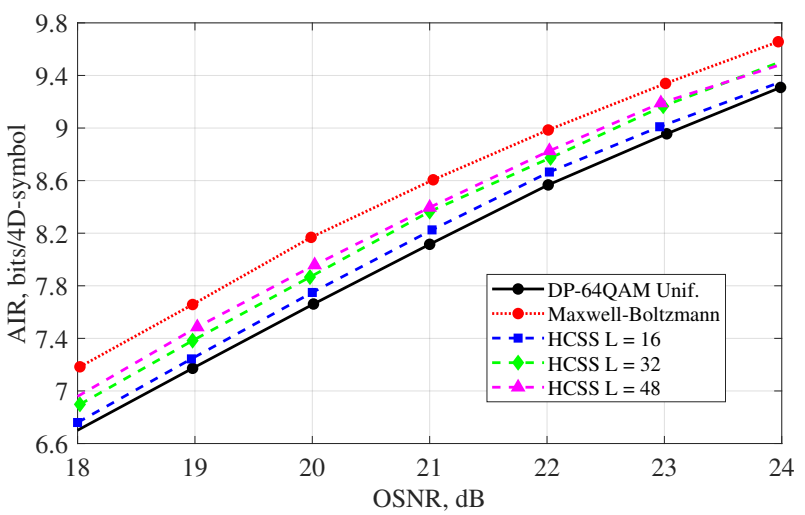

(a)

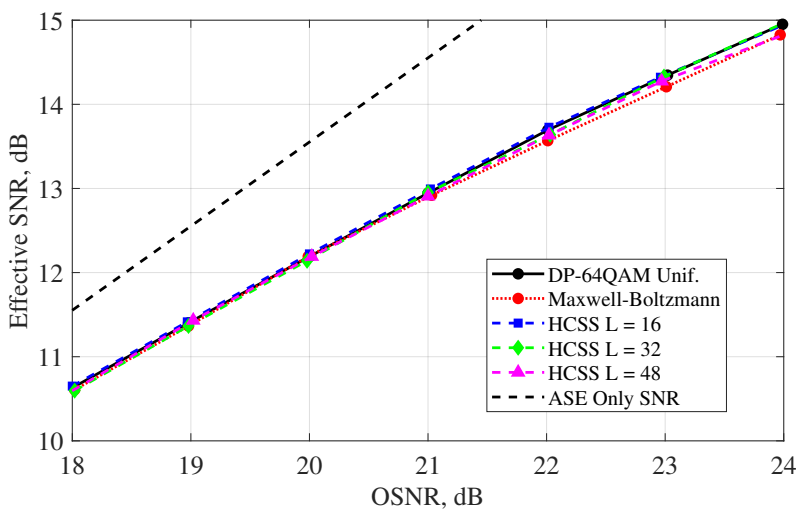

(b)

Fig. 3. Performance vs. OSNR in back-to-back configuration: (a) AIR in b/4D, (b) Effective SNR in dB

\section{B. Transmission over $210 \mathrm{~km}$}

Fig. 4 shows the characterization of nonlinear transmission performance over a link comprising a single span of $210 \mathrm{~km}$ SSMF. According to Fig. 4(a), in the linear regime (power per channel below $8 \mathrm{dBm}$ ), AIR performance is consistent with the observation of the BtB measurements. However, in the nonlinear regime, $\mathrm{MB}$ shaping suffers from severe nonlinear impairment, achieving only $0.22 \mathrm{~b} / 4 \mathrm{D}$ AIR gain at the optimum input power, compared with $0.42,0.40$ and $0.30 \mathrm{~b} / 4 \mathrm{D}$ for HCSS with $L=48,32,16$, respectively. The reason for this is apparent in Fig. 4(b), as the peak effective SNR is reduced by more than $0.6 \mathrm{~dB}$ compared with uniform signaling, while HCSS elicits a gain of up to $0.3 \mathrm{~dB}$.

For a linear channel, HCSS with longer shaping sequence length can achieve better power efficiency for a fixed rate, hence providing increased AIR as demonstrated for the BtB case in Fig. 3. However, for a nonlinear channel, shorter shaping sequence lengths provide a higher nonlinear tolerance, as demonstrated by the effective SNR gain with reducing shaping length in Fig. 4(b). By choosing appropriate shaping length, optimal combination of linear and nonlinear gains can be achieved [17]. We note that in the highly nonlinear regime the advantage of shorter shaping length is even more pronounced - the linear gain contribution becomes smaller and nonlinear gain is more significant.

It is expected that optimal shaping length for single-span links with short nonlinear memory is in the range of tens 


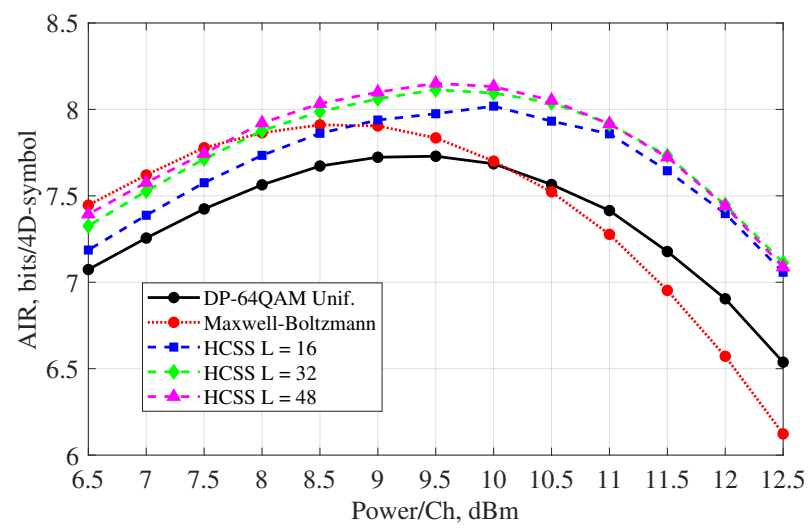

(a)

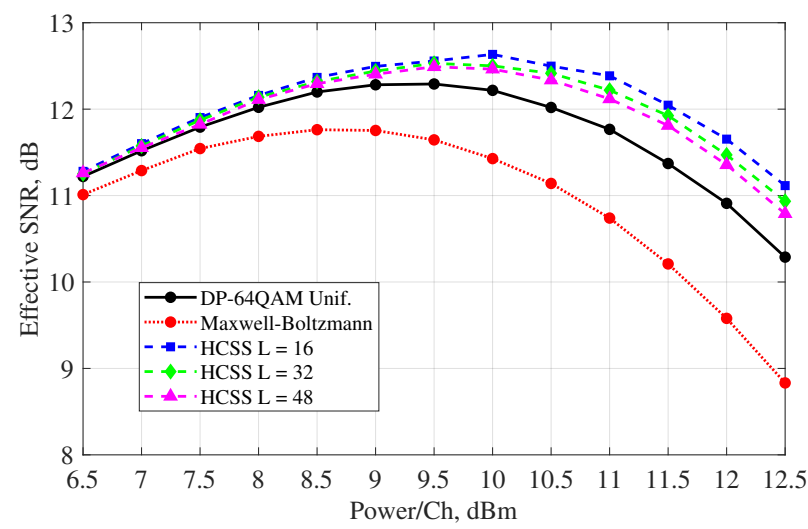

(b)

Fig. 4. Performance vs. optical launch power for transmission over $210 \mathrm{~km}$ : (a) AIR in b/4D, (b) Effective SNR in dB.

of amplitudes for sphere shaping schemes (as theoretically predicted in [17] using shell mapping). For HCSS the optimal shaping sequence length is determined by the trade-off between improved nonlinear tolerance due to shorter shaping length and associated increased rate loss (with corresponding decrease in power efficiency). According to Fig. 4(b), HCSS with $L=16$ provides the highest effective SNR gain of $0.9 \mathrm{~dB}$ over infinite-length MB shaping, however, in terms of AIR it performs worse than longer sequence lengths due to the increased rate loss. While HCSS shaping with $L=32,48$ achieve similar performance, indicating the region of optimal shaping sequence length, $L=32$ may be considered preferable due to its reduced complexity. We note that as $L$ increases HCSS performance approaches that of MB shaping.

\section{Conclusions}

In this paper, the improved nonlinear tolerance of HCSS for extended-reach single-span links was characterized. Shortlength HCSS provides both linear gain, and improved effective SNR in the nonlinear region, enabling superior performance over infinite-length MB shaping. For transmission over $210 \mathrm{~km}$, an AIR gain of $0.4 \mathrm{~b} / 4 \mathrm{D}$ over uniform signaling and $0.18 \mathrm{~b} / 4 \mathrm{D}$ over MB shaping was achieved, using a shaping sequence length of $L=32$, with a corresponding improvement in effective SNR of $0.2 \mathrm{~dB}$ and $0.8 \mathrm{~dB}$, respectively. We note that HCSS algorithms may be implemented without multiplications, and with modest LUT sizes, e.g., for $L=32$ the size is no more than $100 \mathrm{kbit}$.

\section{ACKNOWLEDGMENT}

The work of IP and WF was supported by UK EPSRC grant EP/M009092/1. We thank Lumentum UK for loan of the CFP2-ACO and studentship support of PS, and Socionext for loan of the DAC/ADC DKs used in this work.

\section{REFERENCES}

[1] "OIF 400G ZR implementation agreement." [Online]. Available: http://oiforum.com/wp-content/uploads/OIF-400ZR-01.0_reduced2.pdf

[2] G. Böcherer, F. Steiner, and P. Schulte, "Bandwidth efficient and rate-matched low-density parity-check coded modulation," IEEE Trans. Commun., vol. 63, no. 12, pp. 4651-4665, 2015.

[3] J. Cho et al., "Trans-Atlantic field trial using probabilistically shaped 64 QAM at high spectral efficiencies and single-carrier real-time $250-\mathrm{Gb} / \mathrm{s}$ 16-QAM," in Proc. Opt. Fiber Commun. Conf. (OFC), Los Angeles, CA, USA, 2017.

[4] J. Li et al., "Field trial of probabilistic-shaping-programmable real-time 200-Gb/s coherent transceivers in an intelligent core optical network," in Proc. Asia Commun. and Photon. Conf. (ACP), Guangzhou, China, 2018.

[5] F. Buchali et al., "Rate adaptation and reach increase by probabilistically shaped 64-QAM: An experimental demonstration," J. Lightw. Technol., vol. 34, no. 7, pp. 1599-1609, 2016.

[6] T. Fehenberger et al., "Multiset-partition distribution matching," IEEE Trans. Commun., vol. 67, no. 3, pp. 1885-1893, 2019.

[7] Y. C. Gültekin et al., "Constellation shaping for IEEE 802.11," in Proc. IEEE PIMRC, Montreal, QC, Canada, 2017.

[8] Y. Koganei et al., "Optimum bit-level distribution matching with at most $\mathrm{O}\left(N^{3}\right)$ implementation complexity," in Proc. Opt. Fiber Commun. Conf. (OFC), San Diego, CA, USA, 2019.

[9] T. Yoshida, M. Karlsson, and E. Agrell, "Hierarchical distribution matching for probabilistically shaped coded modulation," J. Lightw. Technol., vol. 37, no. 6, pp. 1579-1589, 2019.

[10] T. Fehenberger and A. Alvarado, "Analysis and optimisation of distribution matching for the nonlinear fibre channel," arXiv:1907.02846, 2019.

[11] T. Fehenberger et al., "On probabilistic shaping of quadrature amplitude modulation for the nonlinear fiber channel," J. Lightw. Technol., vol. 34, no. 21, pp. 5063-5073, 2016.

[12] J. Renner et al., "Experimental comparison of probabilistic shaping methods for unrepeated fiber transmission," J. Lightw. Technol., vol. 35, no. 22, pp. 4871-4879, 2017.

[13] T. Fehenberger, H. Griesser, and J.-P. Elbers, "Mitigating fiber nonlinearities by short-length probabilistic shaping," in Proc. Opt. Fiber Commun. Conf. (OFC), San Diego, CA, USA, 2020.

[14] T. Fehenberger et al., "Analysis of nonlinear fiber interactions for finitelength constant-composition sequences," J. Lightw. Technol., vol. 38, no. 2 , pp. 457-465, 2020.

[15] S. Goossens et al., "First experimental demonstration of probabilistic enumerative sphere shaping in optical fiber communications," in Proc. Opto-Electron. Commun. Conf. (OECC) and Int. Conf. on Phot. in Switch. and Comp. (PSC), Fukuoka, Japan, 2019.

[16] A. Amari et al., "Introducing enumerative sphere shaping for optical communication systems with short blocklengths," J. Lightw. Technol., vol. 37, no. 23, pp. 5926-5936, 2019.

[17] O. Geller et al., "A shaping algorithm for mitigating inter-channel nonlinear phase-noise in nonlinear fiber systems," J. Lightw. Technol., vol. 34, no. 16, pp. 3884-3889, 2016.

[18] D. S. Millar et al., "Huffman coded sphere shaping with short length and reduced complexity," in Proc. Europ. Conf. Opt. Commun. (ECOC), Dublin, Ireland, 2019.

[19] R. F. H. Fischer, Precoding and signal shaping for digital transmission. New York, New York, USA: John Wiley \& Sons, 2005.

[20] T. Fehenberger et al., "Huffman-coded sphere shaping and distribution matching algorithms via lookup tables," J. Lightw. Technol., vol. 38, no. 10 , pp. 2826-2834, 2020.

[21] P. Skvortcov et al., "Receiver DSP highly tolerant to transmitter IQ impairments," in Proc. Opt. Fiber Commun. Conf. (OFC), San Diego, CA, USA, 2019.

[22] A. Alvarado et al., "Achievable information rates for fiber optics: Applications and computations," J. Lightw. Technol., vol. 36, no. 2, pp. 424-439, 2018. 